Revue d'histoire de l'enfance « irrégulière »

Le Temps de l'histoire

$20 \mid 2018$

Sexualités juvéniles

\title{
Errance et abandon : La recirculation des enfants pauvres de Dijon
}

Christopher R. Corley

Traducteur : Sonya Malaborza

\section{CpenEdition \\ Journals}

Édition électronique

URL : http://journals.openedition.org/rhei/4443

DOI : $10.4000 /$ rhei. 4443

ISSN : $1777-540 \mathrm{X}$

Éditeur

Presses universitaires de Rennes

Édition imprimée

Date de publication : 15 novembre 2018

Pagination : 201-218

ISBN : 978-2-7535-7571-4

ISSN : 1287-2431

\section{Référence électronique}

Christopher R. Corley, «Errance et abandon : La recirculation des enfants pauvres de Dijon », Revue d'histoire de l'enfance « irrégulière » [En ligne], 20 | 2018, mis en ligne le 15 novembre 2020, consulté le 04 décembre 2020. URL : http://journals.openedition.org/rhei/4443; DOI : https://doi.org/10.4000/ rhei. 4443 


\section{Errance et abandon : la recirculation des enfants pauvres de Dijon}

\begin{abstract}
Des recherches récentes sur les familles européennes du début des temps modernes ont souligné le caractère malléable de ces familles, de même que la mobilité frappante des populations de l'époque préindustrielle. II semblerait que les ménages reconstitués à la suite du décès ou du départ d'un parent étaient beaucoup plus fréquents que l'on aurait pu le croire. La circulation des jeunes est une composante importante de ces états de fait. Un examen des jeunes circulant autour de I'hôpital général de Dijon au xvIII siècle nous permet d'observer l'émergence de la doctrine in loco parentis au sein des populations préindustrielles. Ce mouvement de jeunes orphelins, abandonnés ou errants, n'est pas nécessairement indicateur d'un échec de la part des familles. À n'en pas douter, les systèmes mis en place pour surveiller et faire circuler ces jeunes constituaient des initiatives collectives capables de tenir compte à la fois des intérêts des plus pauvres familles de Dijon et des résidents les plus privilégiés de la ville.
\end{abstract}

Recent scholarship on the history of the family in premodern Europe has emphasized the malleability of families as well as the striking mobility of preindustrial populations. Reconstituted households caused by parental death or abandonment were far more common than we have previously thought. The circulation of youth formed an important component of these phenomena. An examination of youth who moved in and out of the General Hospital of Dijon in the eighteenth century reveals the emergence of in loco parentis concepts in preindustrial societies. The circulation of orphaned, abandoned, and vagrant youth was not necessarily an indication of systemic failures of families. Instead, we can also see that the systems created for the supervision and circulation of youth were shared initiatives that flexibly accommodated the interests of Dijon's poorest families and most prominent residents.

Mots-clés : enfance, jeunesse, enfance abandonnée, orphelins, mobilité, abandon, hôpitaux généraux, Bourgogne, $\mathrm{xVII}$ siècle.

Keywords: childhood, youth, abandoned children, orphans, mobility, abandonment, orphanage, Burgundy, eighteenth century.

\section{Christopher \\ R. Corley \\ Professeur d'histoire \\ à la Minnesota State \\ University, Mankato, \\ États-Unis. II enseigne \\ l'histoire européenne \\ depuis la Grèce et \\ la Rome antiques \\ jusqu'à la Révolution \\ française, de même \\ que l'histoire des \\ femmes, de la famille \\ et des enfants. Ses \\ recherches portent sur \\ le statut et le quotidien \\ des jeunes pendant \\ l'ancien régime.}

La recherche asso-

ciée à cet article et

sa traduction par

Sonya Malaborza

ont été rendues

possibles grâce à

l'appui de l'École des

études supérieures

et de la recherche

du Minnesota State

University, Mankato. 
1. Archives départementales de la Côte-d'Or (ADCO), 118 H/R art. 1250/7, Registre des enfants à Bonnets Rouges, 02/12/1753

2. LAMARRRE Christine, L'Hôpital de Dijon au xvIII siècle, Langres, Dominique Guéniot, 2004; Garnot Benoît, Vivre en Bourgogne au XVIII siècle, Dijon, Éditions universitaires de Dijon, 1996, p. 57-64; Nelson Jessica, "Policy and Sentiment: Attitudes and Institutions Concerning Abandoned Children in Seventeenthand Eighteenth-century France" (thèse de doctorat), Lafayette, Purdue University, 2014. Sur les pauvres et le rôle des hôpitaux de façon plus générale, voir Hufton Olwen, The Poor of Eighteenth-Century France, 1750-1789,

Oxford, Clarendon Press, 1974 ; Hıckey Daniel, Local Hospitals in Ancien Régime France: Rationalization, Resistance, and Renewal, 1530-1789, Montréal, McGill-Queen's University Press, 1997 ; et Schwartz Robert, Policing the Poor in Eighteenth-Century France, Chapel Hill, University of North Carolina Press, 1988.

3. Fauve-Chamaux Antoinette, "Beyond Adoption: Orphans and Family Strategies in Pre-Industrial France", History of the Family, $\mathrm{n}^{\circ} 1$, 1996, p. 1-13
Dar un froid matin de dimanche en décembre 1753, Claude Garnier, 17 ans, et son compagnon Jean Robert dormaient dans la chapelle de l'hôpital général de Dijon. Garnier était natif de l'Auxois, une région située à une cinquantaine de kilomètres à l'ouest de Dijon, où les fermiers peinaient à travailler les terres des vallées et des collines ondulées. Ce matin-là, lorsqu’il fut intercepté, le jeune homme confia aux autorités qu'il avait dû quitter la maison à cause de son nouveau beau-père et qu'il s'était aventuré jusqu'à la banlieue de Dijon, à l'est, où un journalier l'avait embauché comme assistant. Une fois ce travail épuisé, il avait dû se contenter d'œuvrer comme porteur, transportant du bois pour les ménages qui se préparaient à l'hiver. Depuis trois semaines, il cherchait des lieux où passer la nuit en compagnie de son ami Robert et, plus récemment, avec d'autres garçons qu'il connaissait moins bien ${ }^{1}$. Les autorités de Dijon croisaient parfois par hasard ou lors d'opérations policières de jeunes orphelins, abandonnés ou errants comme Garnier et Robert. Leur solution était de les confier à un établissement voué au soin des pauvres, des malades et des démunis de la ville : l'hôpital général ${ }^{2}$. La direction et le personnel de cet établissement étaient alors chargés de répondre provisoirement aux besoins de ces enfants en les nourrissant et en leur donnant notamment des habits, un abri et une instruction limitée. Tôt ou tard, cependant, il fallait trouver à ces jeunes gens une maison d'accueil où habiter, parfois jusqu'à trois ans, en attendant qu'ils puissent subvenir eux-mêmes à leurs besoins.

Normalement, c'est à la famille proche ou élargie que revenait la garde d'un orphelin d'âge mineur, responsabilité que surveillait dans une certaine mesure le système judiciaire local. Quand le réseau familial et social ne suffisait pas, la famille ou un juge pouvait confier les enfants à des amis proches. Dans certains cas, en milieu rural surtout, un tuteur vendait les services d'un enfant aux enchères publiques. Le soumissionnaire retenu s'engageait alors à prendre soin de l'enfant en espérant en retour le faire travailler dans sa ferme ou son atelier ${ }^{3}$. Malgré tout, beaucoup de jeunes comme Claude Garnier se sont trouvés en marge de ce système improvisé de surveillance et d'éducation. Un chercheur d'une autre époque aurait dit de ces tristes exemples qu'il s'agissait de cas exceptionnels et affirmé que la vaste majorité des enfants étaient pris en charge par leur famille. Or, il s'agit là d'un mythe concernant la cohésion et la stabilité des familles de l'ère préindustrielle qui continue de persister dans l'imaginaire des gens. Les ménages reconstitués à la suite du décès ou du départ d'un parent 
étaient beaucoup plus fréquents que l'on pourrait le croire : des recherches récentes sur les familles européennes du début des temps modernes ont souligné le caractère malléable de ces familles, de même que la mobilité frappante des populations de l'époque préindustrielle ${ }^{4}$. La circulation d'enfants est un aspect important de ces phénomènes. Selon Joel Harrington :

Loin de représenter une anomalie ou un échec du système, la circulation 《informelle d'enfants sous diverses formes constituait un des mécanismes centraux unissant les premières communautés modernes et donc la société dans son ensemble .»

Le sort d'innombrables jeunes filles et garçons miséreux comme Garnier et Robert est un exemple important mais peut-être plus sombre de la circulation de jeunes et du développement du concept d'in loco parentis dans les premières sociétés modernes. À n'en pas douter, la supervision et la circulation des jeunes orphelins, abandonnés ou errants permettait non seulement de tenir compte à la fois des intérêts des plus pauvres familles de Dijon et des résidents les plus privilégiés de la ville, mais aussi de répondre aux besoins économiques des familles des paysans pauvres qui peuplaient la campagne bourguignonne.

\section{LES HÔPITAUX, LA SÉCURITÉ SOCIALE ET L'ABANDON D'ENFANTS}

\section{À L'ÈRE PRÉINDUSTRIELLE}

La prise en charge des enfants miséreux laissés aux soins d'une institution a pris de l'essor vers la fin du Moyen-Âge. En effet, l'apparition des premiers orphelinats remonte au $\mathrm{XV}^{\mathrm{e}}$ siècle en Europe méridionale, et ces établissements se sont disséminés vers le nord aux XVII ${ }^{\mathrm{e}}$ et XVIII ${ }^{\mathrm{e}}$ siècles. Dans leur enceinte, les enfants apprenaient à lire et à écrire dans un environnement très réglementé, conçu de façon à inculquer des valeurs d'humilité et une éthique du travail. En grandissant, certains enfants pouvaient se voir offrir un apprentissage, une dot ou une autre forme d'aide. Quelques chanceux étaient adoptés ${ }^{6}$; d'autres étaient expédiés vers un avenir incertain dans les colonies.

Les $\mathrm{XVII}^{\mathrm{e}}$ et XVIII ${ }^{\mathrm{e}}$ siècles ont vu une expansion massive des hôpitaux et des orphelinats. Dans ces établissements, de plus en plus de laïques surveillaient les activités des religieux et des religieuses chargées des opérations journalières. Après 1660 , la protection des «enfants de l'État » a retenu l'attention des

\author{
4. Desan Suzanne, MerRick \\ Jeffrey (dir.), Family, \\ Gender, and Law in Early \\ Modern France, University \\ Park, Pennsylvania \\ State University Press, \\ 2009; Dos Guimaraes Sa \\ Isabel, "Circulation of \\ Children in Eighteenth- \\ Century Portugal", in \\ PANTER-Brick Catherine et \\ SMITH Malcolm T. (dir.), \\ Abandoned Children, \\ Cambridge, Cambridge \\ University Press, 2000, \\ p. 27-40. \\ 5. HARRINGTON Joel, The \\ Unwanted Child: The Fate of \\ Foundlings, Orphans, and \\ Juvenile Criminals in Early \\ Modern Germany, Chicago, \\ University of Chicago Press, \\ 2009, p. 278. \\ 6. Gager Kristin, Blood Ties \\ and Fictive Ties: Adoption \\ and Family Life in Early \\ Modern France, Princeton, \\ Princeton University Press, \\ 1996.
}


7. LamarRe, L'Hôpital de Dijon..., op. cit., p. 1-95; HICKEY, Local Hospitals, op. cit., p. 102; Pullan Brian,

"Catholics, Protestants, and the Poor in Early Modern Europe", Journal of Interdisciplinary History, no 35, 2005, p. 441-456; Viazzo Pier Paolo, "Mortality, Fertility, and Family", in Kertzer David et Barbagl Marzio (dir.), The History of the Family, Volume I: Family Life in Early Modern Times, New Haven, Yale University Press, 2001, p. 186-187.

8. Delaselle Claude, " Les enfants abandonnés à Paris

au xIII siècle ", Annales. Economies, Sociétés, Civilisations, 1, 1975, p. 188.

9 Hufton, The Poor of Eighteenth-Century France, op. cit., p. 318.

10. LAMARRE Christine, « La vie des enfants et des vieillards assistés à Dijon au XVIII ${ }^{e}$ siècle », European University Institute Working Papers nos 89-403, Florence, European University Institute, 1989, p. 34. autorités politiques; il s'agissait, après tout, d'un prolongement logique des politiques pro-nationalistes et paternalistes du gouvernement. Les historiens parlent de l'âge d'or des orphelinats en Europe et dans les Amériques.

$\mathrm{Au} \mathrm{XVIII}{ }^{\mathrm{e}}$ siècle, on compte en Bourgogne 52 établissements chargés des enfants malades, infirmes, abandonnés ou orphelins de la région. Les services offerts aux plus fragiles et aux plus miséreux étaient assurés par des religieux et des employés rémunérés. Très différent de l'établissement de soins d'aujourd'hui, l'hôpital de l'ère préindustrielle était un lieu de derniers recours; la plupart des gens fortunés pouvaient compter sur leur famille et leurs amis et s'offrir les services d'un médecin à domicile. La surveillance administrative variait de lieu en lieu, mais la gestion des politiques et des finances de l'hôpital de Dijon était confiée aux juges et aux fonctionnaires dont le travail les amenait à affronter les conséquences de la pauvreté, du crime et du désordre social7

Partout en Europe continentale, au cours du siècle qui précède la Révolution française, de plus en plus d'enfants se sont retrouvés devant la porte d'hôpitaux, et leur nombre dépassait de loin le rythme de croissance de la population en général. Selon une étude de la situation à Paris, le nombre d'enfants admis à un des orphelinats est passé de 1700 par année au début du XVIII ${ }^{\mathrm{e}}$ siècle à 7676 en $1772^{\circ}$. Olwen Hufton estime quant à lui que près de 40000 enfants français étaient abandonnés chaque année vers la fin de l'Ancien Régime?.

L'infrastructure sociale et politique de l'époque étant mal équipée pour résoudre ce problème, le taux de survie des nourrissons abandonnés est déplorable. À Rennes, une ville située dans l'ouest de la France, seulement $20 \%$ des enfants abandonnés vers la fin du XviII ${ }^{\mathrm{e}}$ siècle survivaient plus de deux mois. Le taux de survie à l'Hôtel-Dieu de Paris entre 1772 et 1778 était encore plus fâcheux : $17 \%$. La situation à Dijon était meilleure, mais les statistiques peignent tout de même un portrait sombre. Christine Lamarre estime que 840 des 1954 enfants recueillis par l'hôpital entre 1752 et 1771 sont morts avant l'âge de 12 ans $^{10}$. Les heureux survivants étaient confrontés à une enfance difficile : ils devaient changer de maison d'accueil et de village aux deux ou trois ans, passant brièvement à l'orphelinat où on leur transmettait les connaissances de base dont ils avaient besoin pour vivre de façon autonome vers le milieu ou la fin de l'adolescence.

Pour venir en aide à ce nombre croissant d'enfants abandonnés, beaucoup d'hôpitaux, craignant une insuffisance de ressources, ont dû adopter des 
approches novatrices. Même les efforts conjugués de l'Église, de la Couronne et des villages environnants ne suffisaient plus à la demande toujours croissante. En 1746, l'hôpital de Dijon nourrissait à lui seul près de 2000 enfants, personnes âgées, malades et infirmes. Les responsables du milieu hospitalier et du gouvernement préconisaient d'envoyer les enfants à la campagne. Cette solution a d'abord été évoquée à Dijon en 1681, sans grand effet. En 1723, l'hôpital de Dijon a suivi cette recommandation en plaçant les nourrissons ayant été laissés - souvent au milieu de la nuit - aux portes de la ville ou devant une église, une chapelle ou l'hôpital. En 1748, tous les enfants recueillis de moins de 12 ans étaient placés à la campagne ${ }^{11}$. Charles de Brosses, un directeur de l'hôpital, offrait la justification suivante :

L'air ouvert de la campagne leur conserve la santé, non seulement conserve 《une certaine quantité de citoyens précieux à l'état, mais de plus leur forme un tempérament robuste et les accoutume dès la première jeunesse à l'habitude et au goût des travaux de la campagne $e^{12}$."

Nonobstant cette justification, il est clair que la situation financière de l'hôpital y était pour quelque chose. De Brosses estime que l'hôpital devait débourser 6039 livres par année pour s'occuper de 100 enfants, ce qui, à la campagne, ne coûterait que 3600 livres (à 3 livres par mois par enfant, ou 36 livres par année). La croyance générale voulait qu'accueillir chez soi un enfant était très profitable. Comme le souligne de Brosses, le nombre de familles prêtes à accueillir des enfants était supérieur au nombre d'enfants à loger. Une bouche de plus à nourrir ne mettait pas vraiment le budget familial à l'épreuve, et les sommes obtenues pouvaient servir à payer les dépenses importantes. La direction espérait que les enfants de plus de 12 ans continueraient d'être logés à peu de frais dans leur foyer d'accueil, qu'ils acquerraient des compétences et qu'ils finiraient par gagner leur vie dans la campagne bourguignonne. En 1761, on voit apparaître une autre mesure incitative quand la monarchie déclare que tout enfant de plus de 16 ans logé par une famille d'accueil dispenserait cette famille d'envoyer un fils ou un neveu en service militaire. L'enfant placé chez eux pouvait prendre sa place dans les rangs ${ }^{13}$.

Malgré les efforts déployés pour envoyer les jeunes de 12 ans et plus à la campagne, quelques enfants plus âgés devaient rester sous la garde de l'hôpital jusqu’à la fin de l'adolescence. En effet, une quarantaine de garçons et une
11. Bolotte Marcel, Les hôpitaux et l'assistance dans les provinces de Bourgogne au dernier siècle de l'Ancien Régime, Montpellier, Imprimerie universitaire, 1968, p. 36; LAMARRE, L'Hôpital de Dijon, op. cit., p. 269-270.

12. Bibliothèque municipale de Dijon (BMD), ms. 625, Joly de Bevy, " Histoire de l'hôpital du Saint-Esprit et Nôtre-Dame de la Charité de la Ville de Dijon », p. 145.

13. BMD, ms. 625 , p. $160-161$. 
14. ADCO, $118 \mathrm{H} / \mathrm{R}$ art. 1250/7, Registre des enfants à Bonnets Rouges, 1706-1820; ADCO H dep. 239/1/F, art. 2-1, Filles de Sainte-Anne, 1713-1820.

15. Morlot Sophie, Enfants et enfances dans le Dijon révolutionnaire et consulaire [thèse de doctorat, 2 vols.], Dijon, université de Bourgogne, 2008, vol. 1, p. 490.

16. Nelson Jessica, "Gender, Age, and the Abandonment of Children in EighteenthCentury Dijon", Journal of the History of Childhood and Youth, n० 4, 2011, p. 116-135.

17. FucHs Rachel, Abandoned Children: Foundlings and Child Welfare in NineteenthCentury France, Albany, State University of New York Press, 1984, p. 1 centaine de filles, dont beaucoup souffrant de problèmes de santé à long terme ou d'un handicap quelconque, auraient eu beaucoup de mal à vivre de leur travail. Parmi tous les établissements chargés de surveiller ces enfants, deux registres d'admissions ont été conservés : celui des Bonnets Rouges et celui des Filles de Sainte-Anne ${ }^{14}$. Ces documents nous permettent de voir comment se sont effectuées les transitions, chez un petit segment des enfants abandonnés, depuis la petite enfance jusqu'à la fin de l'adolescence et le début de l'âge adulte. S’il est vrai que nous ne saurons rien de la plupart des expériences vécues par les enfants abandonnés après 12 ans, les données que referment ces deux registres jettent un peu de lumière sur les expériences vécues par un groupe unique et nous donnent des indices quant aux expériences partagées par la vaste majorité des enfants abandonnés. On ne peut pas tirer de ces registres des statistiques précises sur le sort de ces jeunes ${ }^{15}$, mais nous y trouvons tout de même des pistes quant aux raisons pour lesquelles les enfants ont été recueillis, de même que de l'information sur les caractéristiques de leur séjour à l'hôpital, leur intégration sociale et leur transition à la vie adulte ${ }^{16}$.

\section{LES MÉNAGES DE DIJON ET SES HÔPITAUX : LES CAUSES DE L'ABANDON}

L'abandon d'enfants est une triste réalité de l'histoire européenne, et les causes et les modalités qui la sous-tendent sont complexes. Comme l'a montré Christine Lamarre, les parents empruntaient l'une des trois méthodes pour abandonner un enfant : ils le laissaient dans un lieu public (exposition), le présentaient à l'hôpital par l'entremise d'un tiers (une sage-femme ou un médecin, par exemple), ou adressaient eux-mêmes une requête à l'hôpital. S'il est vrai qu'exposer un enfant dans un lieu public est considéré comme un crime en France depuis 1556, l'action était jugée moins grave que l'infanticide. Devant le nombre croissant d'enfants abandonnés dans la région, l'hôpital de Dijon a fait construire son tour, une armoire tournant sur pivot placée dans l'épaisseur d'un mur dans laquelle se trouvait un berceau. Ce faisant, on souhaitait inciter les mères à abandonner leur nourrisson dans un endroit plus sûr que sous un pont, à la porte d'une ville ou devant une église, un monastère ou un couvent ${ }^{17}$.

Le moyen d'abandon le plus socialement acceptable était de passer par un tiers parti pour présenter une demande d'accueil ou bien de présenter soi-même 
cette demande en tant que parent. Après 1750, il est devenu plus commun pour une sage-femme ou un médecin de présenter un enfant à l'hôpital de Dijon peu après sa naissance. La direction supposait alors qu'il s'agissait d'un enfant illégitime dont la mère, domiciliée à Dijon, était trop miséreuse pour s'en occuper convenablement, risquant de perdre son poste et de se faire humilier publiquement pour avoir mis au monde un enfant bâtard ${ }^{18}$. Les parents pouvaient également s'adresser directement à l'hôpital en demandant d'accueillir provisoirement leur enfant en raison de leur pauvreté, d'un handicap physique de l'enfant ou d'autres problèmes d'ordre médical. Si un enfant avait été abandonné pour cause de pauvreté ou de maladie, il avait normalement passé le cap de la petite enfance et avait même parfois atteint l'adolescence. L'hôpital pouvait également accueillir un enfant de manière provisoire pour donner du répit à une famille en difficulté. C'est ce qui est arrivé au maçon Jean Tiguet après la mort de son épouse. Ce dernier s'est adressé aux administrateurs municipaux de Dijon pour leur informer qu'il n'avait pas de logement et très peu de possessions matérielles; ses trois enfants ont donc été accueillis à l'orphelinat de l'hôpital ${ }^{19}$. Les soins à court et à long termes constituaient des actes de bienfaisance envers les pauvres. Comme on peut l'imaginer, il existe de nombreux cas où la négligence des parents sous-tendait l'abandon des enfants. Certains parents abandonnaient tout simplement leurs enfants dans les rues de la ville sans la moindre intention de retourner les chercher ${ }^{20}$.

Les multiples scénarios ayant mené à la décision de parents d'abandonner leurs enfants nous laissent entrevoir quelques causes possibles. Le recul de l'âge du mariage et la faiblesse de l'espérance de vie, par exemple, faisaient en sorte qu'un enfant allait probablement perdre au moins un parent avant l'âge de 25 ans. La plupart des ménages pauvres comptaient sur les salaires pour subvenir à leurs besoins quotidiens, et la perte du revenu du père pouvait anéantir un budget familial déjà précaire ${ }^{21}$. Le XviII ${ }^{\mathrm{e}}$ siècle a vu une hausse du nombre d'enfants illégitimes en raison notamment du recul de l'âge de mariage. Les mères seules et miséreuses sans autre recours pouvaient chercher à s'épargner l'humiliation et la détresse économique en se servant de l'hôpital comme dispositif de protection ${ }^{22}$. La guerre, la maladie, les famines fréquentes et autres crises de subsistance ont contraint bien des parents et des proches, surtout ceux des échelons inférieurs, à abandonner leurs enfants dans les temps difficiles ${ }^{23}$. Les sources dont nous disposons ne permettent pas vraiment de chiffrer ces

\section{Ibid., p. 104 \\ 19. Archives municipales de Dijon, L 40, 19/09/1729. \\ 20. BMD ms. 625, Joly de Bevy, « Histoire de l'hôpital du Saint-Esprit et Notre- Dame de la Charité de la Ville de Dijon », cité dans LamarRe, L'Hôpital de Dijon, op. cit., p. 106.}

21. Schwartz, Policing the Poor, op. cit., p. 109.

22. Voir par exemple ADCO, B2 360 210, 11/09/1731, où I'on peut lire le témoignage de la jeune domestique Gabrielle Levesque sur la violence sexuelle qu'elle a subie aux mains de son maître. Lorsqu'elle s'est retrouvée enceinte, son maître lui a dit qu'il paierait pour nourrir l'enfant si elle accouchait d'un garçon. Si, toutefois, elle devait accoucher d'une fille, l'enfant serait confié à l'hôpital.

23. BARDET Jean-Pierre, FARON Olivier, "Des enfants sans enfance : sur les abandonnées de l'époque moderne ", in BЕCCHI Egle, JulıA Dominique (dir.), Histoire de l'enfance en occident, Paris, Seuil, 1998, p. 139; HaRRINGTON, The Unwanted Child, p. 286-289 Gerber Matthew, Bastards: Politics, Families, and Law in Early Modern France, Oxford, p. 135-143. 
24. BMD ms. 625, Joly de Bevy, « Histoire de l'hôpital du Saint-Esprit », cité dans LAMARRE, L'Hôpital de Dijon au XVIIF siècle, op. cit., p. 106

25. Nelson, « Gender, Age... », op. cit., p. 124

26. ADCO $118 \mathrm{H} / \mathrm{R}$ art 1250/7, f. 58r., 23/02/1755

27. Voir, par exemple, ADCO $118 \mathrm{H} / \mathrm{R}$ art. 1250/7, f. 41r., 27/09/1750; f. 43r., 05/07/1751; f. 56v. 15/09/1754; f. 90v., 03/03/1770; f. 110v. 21/07/1770

28. Dinet-Lecomte MarieClaude, « Les enfants de la misère à l'hôpital. Les 5-25 ans à l'hôpital général de Blois de 1752 à 1812 »,

Annales de démographie historique, $\mathrm{n}^{\circ} 20,1991$ p. 203-219; Beauvalet-

Boutouyrie Scarlett, « Les enfants de la Salpêtrière au XVIII ${ }^{e}$ siècle », p. 888 ; BARDET Jean-Pierre et al.

(dir.), Lorsque l'enfant grandit : entre dépendance et autonomie, Paris, Presses

de l'Université de Paris-

Sorbonne, 2003, p. 888 ;

Robin-ROMERo Isabelle

«Les établissements pour orphelins à Paris aux $\mathrm{XV} I^{\mathrm{e}}-\mathrm{XVIII}{ }^{\mathrm{e}}$ siècles », Histoire, économie et société, n 17 , 1998, p. 446. situations, mais plusieurs documents de l'époque offrent des descriptions de parents ayant simplement laissé leur enfant sur le pavé. La négligence parentale était donc parfois une cause probable d'abandon ${ }^{24}$.

Les données recueillies à Dijon corroborent en grande partie ces scénarios. Bon nombre des adolescents répertoriés dans les registres des Bonnets Rouges et des Filles de Sainte-Anne ont d'abord été abandonnés à la petite enfance et ont séjourné dans ces établissements afin d'y être instruits ou soignés, ou encore en attendant d'être envoyés dans une nouvelle famille d'accueil. D'autres enfants ont été abandonnés ou sont devenus orphelins plus tard. L’analyse qu'a faite Jessica Nelson des registres de la première moitié du siècle montre que pour plus de la moitié des enfants recueillis à l'hôpital, on ne connaissait rien des parents, ces enfants ayant été abandonnés de façon anonyme peu après leur naissance. Dans les cas où le contexte familial était connu, « la plupart des enfants venaient de familles pauvres de la classe ouvrière »; les parents étaient journaliers, artisans pauvres ou cultivateurs de raisin ${ }^{25}$.

Beaucoup d'adolescents accueillis à l'hôpital n'étaient pas à proprement parler orphelins. Il arrivait qu'un jeune dont les parents venaient d'être incarcérés soit envoyé à l'hôpital par les autorités judiciaires ${ }^{26}$. D'autres étaient atteints d'un handicap ou d'une maladie de longue durée; cette cause importante d'abandon mérite d'ailleurs qu'on y accorde un plus grand intérêt. Bon nombre d'enfants avaient des parents qui étaient eux-mêmes hospitalisés en raison d'une maladie grave ${ }^{27}$. Des études menées par Marie-Claude Dinet-Lecomte et Scarlett Beauvalet-Boutouyrie sur les enfants abandonnés à Blois et à Paris confirment cette tendance. Les adolescents, écrit Beauvalet-Boutouyrie, « [étaient] surtout les enfants malades ou physiquement déficients, ainsi que ceux qui [n'étaient] pas parvenus à rester en apprentissage à l'extérieur ». Beauvalet-Boutouyrie fait d'ailleurs valoir que la misère dépassait la maladie comme principale cause d'abandon au XvIII ${ }^{\mathrm{e}}$ siècle, les enfants dont les parents étaient toujours vivants ayant passé de $26 \%$ des personnes admises à l'hôpital dans les années 1720 à $77 \%$ dans les années $1780^{28}$.

Le pourcentage élevé d'enfants dont les parents étaient toujours vivants, de pair avec des mentions brèves de ces jeunes dans les registres d'admissions, porte à croire que certaines formes d'abandon étaient considérées comme provisoires. Des témoignages de l'époque appuient cette hypothèse. Joly de Bévy l'explique ainsi : 
La charité des intendants de l'hôpital vient non seulement au secours des 《enfants bâtards et abandonnés, mais elle pourvoit encore à la subsistance des enfants légitimes de familles miséreuses. On admet à l'hôpital sur les requêtes qui sont présentées par les pauvres artisans et autres habitants de la ville et des faubourgs de Dijon seulement, un de leurs enfants et quelquefois deux ${ }^{29}$."

L'hôpital prenait parfois en charge pendant un temps des enfants ayant récemment perdu un parent, même si l'autre était toujours vivant. L'hôpital pouvait accueillir un enfant d'une famille qui en comptait cinq, ou deux enfants si elle en comptait sept ou plus.

Une requête présentée par la famille Benoist en novembre 1745 illustre bien la situation dans laquelle pouvaient se trouver certains ménages et montre à quel point l'hôpital pouvait faire preuve de souplesse à leur égard. Dans une missive, Benigne Benoist et son épouse Estienette Germiniet demandent à la direction de l'hôpital d'accueillir leur fille Nicole, 8 ans, puisqu' « ils se trouvent chargés de trois autres petits enfants et que le père est détenu malade à l'hôpital depuis trois mois ». Après avoir confirmé la situation auprès du curé de la paroisse, les directeurs ont accepté d'accueillir la fillette pendant un an et de faire sortir de l'hôpital la sœur aînée de Nicole, Pierrette Benoist, 12 ans. Les parents n'en étaient pas à leur première interaction avec l'hôpital, et comme c'était souvent le cas, ils ont demandé à faire prolonger le séjour. En fin de compte, Nicole n'allait quitter l'hôpital qu'en avril 1750 , quatre ans plus tard que prévu ${ }^{30}$. De tels exemples d' "échanges " parsèment les registres ${ }^{31}$. En 1754, la direction a constaté que Jeanne Farcy, 10 ans, ne s'adaptait pas à l'hôpital auquel elle venait d'être admise; ses parents l'ont donc échangée avec sa sœur cadette Claudine ${ }^{32}$. Les seigneurs des villages environnants utilisaient des fonds de dotation créés par des générations précédentes dans le but express de venir en aide aux filles pauvres du village, les faisaient circuler entre leur village et Sainte-Anne pendant deux, quatre ou six ans $s^{33}$. D'autres, attristés peut-être par la mort d'une domestique bien-aimée, parrainaient le séjour à l'hôpital de son enfant et sa formation professionnelle ${ }^{34}$. Ces exemples montrent que les hôpitaux servaient à faire circuler les enfants au sein d'établissements et de familles qui agissaient alors in loco parentis, réduisant ainsi le stress financier pendant une période difficile.

On trouve dans les registres des mentions qui confirment que la direction tenait souvent à ce qu'un enfant donné séjourne chez eux de façon temporaire. Et

\author{
29. BMD, ms. 625 , \\ p. $137-138$. \\ 30. ADCO, H dep. 239/1/F, \\ art. 2-1, f. 56v., 29/11/1745 \\ 31. NeLson, « Gender, \\ Age... ", op. cit., p. 124-125. \\ 32. ADCO, H dep. 239/1/F, \\ art. 2-1, f. 80v., 20/01/1754 \\ 33. Voir, à titre d'exemple, \\ ADCO, H dep. 239/1/F, \\ art. $2-1$, f. $124,11 / 08 / 1771$ \\ f. $127,12 / 09 / 1773$. \\ 34. Voir, à titre d'exemple, \\ ADCO, H dep. 239/1/F, \\ art. 2-1, f. 82v, 12/05/1754, \\ et ADCO $118 \mathrm{H} / \mathrm{R}$ art. \\ 1250/7, f. 54v., 21/04/1754, \\ où l'on apprend que Reine \\ Baron, 9 ans, et son frère \\ Claude, 7 ans, ont été reçus \\ à l'hôpital « en considé- \\ ration des charités que \\ Monsieur de Berbisey a \\ fait et fait encore en faveur \\ des pauvres de l'hôpital ". \\ Le père des enfants Baron \\ travaillait comme domes- \\ tique à la résidence des \\ Berbisey.
}


35. Gutton Jean-Pierre,

"L'insertion sociale des enfants recueillis par la charité de Lyon au XVIII siècle : Un bilan provisoire ", BARDET Jean-Pierre et al. (dir.), Lorsque l'enfant grandit, p. 929-939.

36. Au sujet des messages laissés autour du cou d'un nourrisson, voir par exemple

ADCO, $\mathrm{H}$ dep. 239/1/F art. 2-1, f. 78r., 10/09/1753; sur les soupçons de fraude commise par les nouvelles mères, voir BMD, ms. 625

37. ADCO, $118 \mathrm{H} / \mathrm{R}$ art. 1250/7, f. 52v. 8/11/1753. Voir aussi f. 41v, $18 / 01 / 1750$

38. ADCO, H dep. 239/1/F art. 2-1, f. 121, 18/03/1770.

39. $\mathrm{ADCO}, 118 \mathrm{H} / \mathrm{R}$ art. 1250/7, f. 50r. 17/06/1753. Pour d'autres exemples du même ordre voir f. 53r., 04/04/1754, et

f. $54 v ., 15 / 04 / 1754$

40. $\mathrm{ADCO}, 118 \mathrm{H} / \mathrm{R}$ art. $1250 / 7$, f. $76 \mathrm{v}$. $11 / 02 / 1753$ et $07 / 05 / 1754$

41. ADCO, H dep. 239/1/F art. 2-1, f. 78r., 22/08/1753

42. ADCO, $118 \mathrm{H} / \mathrm{R}$ art. $1250 / 7$, f. 113 r. $14 / 08 / 1771$ c'est parfois ce qui se produisait : certaines mères ayant abandonné leur enfant en bas âge avaient clairement l'intention de retourner le chercher. Plusieurs d'entre elles nouaient un message autour du cou de l'enfant où était écrit son prénom ou fixaient un objet identifiable comme un médaillon sur le bras ou dans la main de l'enfant ${ }^{35}$. Il arrivait à la direction de soupçonner une mère de fraude, ayant d'abord abandonné son enfant puis, peu de temps après, s'étant proposée comme nourrice à l'hôpital afin d'être payée pour l'allaiter ${ }^{36}$. On croise de nombreux exemples de parents, presque toujours la mère, qui auront récupéré leur enfant à l'hôpital à une date ultérieure. Des parents ayant déménagé à Paris, par exemple, envoyaient un membre de la famille chercher l'enfant ou se présentaient euxmêmes pour le faire. Charles Martin, laissé sur le seuil de l'hôpital à un jeune âge, a été demandé par sa mère à Paris en novembre 1753, alors qu'il avait 15 ans ${ }^{37}$. Claudine Roger, atteinte de scrofule, a vécu pendant 17 ans dans diverses familles d'accueil avant d'être récupérée par sa mère en $1777^{38}$. La mère de Guillaume Barthelemy Gueniot a retiré son fils de l'hôpital moins d'un an après qu'il y avait été admis suite à la mort de son père; elle voulait lui donner un métier ${ }^{39}$. Après avoir laissé sa fille de 8 ans à la porte d'un couvent en février 1753, Pierrette de Salorney est retournée la chercher en mai de l'année suivante et l'a « reconnu[e] pour son enfant $\mathrm{t}^{40} »$. Ainsi, des parents faisaient circuler de plein gré leurs enfants dans le système hospitalier pour réduire leur fardeau et celui de leur ménage, ne serait-ce que quelques mois.

On ne doit pas s'étonner d'apprendre que les espoirs des directeurs ont souvent été déçus et que certains enfants accueillis provisoirement n'ont jamais été récupérés. En 1753, Elizabeth Verrière a été accueillie par charité dans un hôpital pendant un an, mais quand le temps était venu pour elle de rentrer à la maison, sa mère était introuvable ${ }^{41}$. Benoist Laurent, 7 ans, avec deux de ses frères et sœurs, a été abandonné de la même façon par ses parents en avril 1771. Huit ans plus tard, quand Benoist, alors âgé de 16 ans, se préparait à partir en apprentissage, ses parents n'avaient toujours pas été retrouvés ${ }^{42}$. Certains des parents n'avaient ni la possibilité ni l'intention de récupérer leur enfant, et bon nombre avaient été abandonnés bien après la petite enfance. Il n'était pas rare de voir cette situation se produire avec des parents dont le travail les amenait à devoir voyager souvent. Dans un cas, l'hôpital a attendu le retour des parents de Jacques Dandenot, 12 ans. Le père de ce dernier, soldat, était absent depuis la naissance de l'enfant en 1742 ; quant à sa mère, elle était introuvable. En 
1755, l'hôpital était toujours sans nouvelles des parents ${ }^{43}$. En 1753, un comédien abandonnait sa fille Anne Pacosse, âgée d'environ 9 ans. La direction souhaitait pouvoir garder la fille " jusqu'au retour du père ou de la mère, ou jusqu'à ce qu'on puisse découvrir leur demeure ${ }^{44}$ ». Il n'était pas rare qu'un remariage ou des problèmes de solvabilité mènent à l'abandon d'un enfant. Jacques Legros, 12 ans, a été admis à l'hôpital parce que son père s'était remarié. Ce dernier délaissait Jacques et les autres enfants issus de son mariage précédent et fuyait la ville, ses créditeurs à ses trousses ${ }^{45}$. Quand les parents n'avaient pas du tout l'intention de récupérer leur progéniture, la direction des hôpitaux adoptait une stratégie double. D'abord, on instaurait un régime rigoureux conçu pour inculquer la piété chrétienne et une éthique du travail durant la courte durée du séjour des enfants à l'hôpital. Ensuite, on cherchait des moyens rentables de faire circuler les enfants au sein d'un réseau de familles d'accueil dans la campagne bourguignonne en attendant qu'ils puissent passer à l'étape suivante de leur vie.

\section{L'HÔPITAL COMME ABRI PROVISOIRE : L'ÉDUCATION,}

\section{LA CIRCULATION ET L'INTÉGRATION SOCIALE DES ENFANTS}

\section{ABANDONNÉS EN BOURGOGNE}

De façon générale, la réaction du milieu hospitalier aux traumatismes sociaux et psychologiques vécus aux mains des parents par les enfants abandonnés a été de leur imposer une discipline. Ceux qui séjournaient plus longtemps chez les Bonnets Rouges ou à Sainte-Anne devaient se conformer à un régime rigoureux. Le réveil avait lieu dès $4 \mathrm{~h} 30$, après quoi on accordait aux enfants un peu de temps pour faire leur toilette, s'habiller et prier. L'essentiel de la journée était ensuite passé à travailler dans l'usine de l'hôpital, avec en plus quelques heures vouées à l'éducation (les bases de la lecture et de l'écriture) et au catéchisme (l'apprentissage par cœur de prières et de chants), entrecoupé de deux brèves pauses pour des activités récréatives et ludiques ${ }^{46}$. En imposant tous ces règlements, l'hôpital souhaitait limiter les activités " traditionnelles » de jeunesse; il était notamment interdit de courir, de faire du bruit, de hurler ou de crier (d'autant plus s'il s'agissait d'obscénités), de jouer aux cartes, de consommer de l'alcool ou d'avoir des rapports avec une personne du sexe opposé. Sous l'effet de certains divertissements de prédilection, il était explicitement interdit de jeter des objets par la fenêtre, de grimper au clocher de la chapelle et de
43. ADCO, $118 \mathrm{H} / \mathrm{R}$ art. 1250/7, f. 48v., 07/11/1752; voir aussi ADCO H dep. 239/1/F, art. 2-1, f. 79r., $17 / 12 / 1753$.

44. ADCO, H dep. 239/1/F art. 2-1, f. 82r., 16/09/1753

45. ADCO, $118 \mathrm{H} / \mathrm{R}$ art. 1250/7, f. 48r., 17/12/1752.

46. BMD, ms. 625 , p. 161-178. 
47. Ibid., p. 138-139 Nelson, "Gender, Age... ", op. cit., p. 117-120

48. Nelson, "Gender, Age... », ibid., p. 129 Dinet-Lecomte, dans « Les enfants de la misère à l'hôpital », p. 213, constate une tendance sexospécifique semblable à l'hôpital de Blois. s'aventurer au-delà du terrain de l'hôpital. Si l'on souhaitait quitter l'hôpital, il fallait obtenir l'autorisation expresse de le faire; toute promenade était strictement surveillée. Les enfants désobéissants risquaient de se faire administrer des raclées en public, de recevoir des coups de fouet, d'être enfermés et de voir leurs rations alimentaires limitées. Cette approche avait pour objectif d'inciter les enfants à saisir rapidement l'importance de l'humilité, de l'obéissance et d'une dévotion à Dieu et de reconnaître la place qu'ils allaient devoir occuper dans la société en gagnant leur vie grâce à leur travail d'artisan, de manœuvre ou de domestique. Supposant que les parents n'avaient pas pu ou n'avaient pas voulu agir ainsi, les directeurs se donnaient pour devoir de transmettre ces notions ${ }^{47}$. Le comportement, les bonnes manières et la tenue des enfants, en particulier lors des offices et des processions publiques, étaient autant de manifestations de leur réussite.

Suite à l'adoption de nouvelles politiques en 1748, la période pendant laquelle les enfants pouvaient être instruits et encadrés par l'hôpital a été réduite car jugée trop coûteuse. Selon les registres des hôpitaux, les garçons et les filles étaient accueillis à l'hôpital à 12 ans environ; les garçons y restaient jusqu'à 14 ans et les filles, jusqu'à 15 ou 16 ans environ. L'âge moyen de départ était considérablement en deçà de ce qu'il avait été au début du XvıII ${ }^{\mathrm{e}}$ siècle : après 1750, les filles quittaient l'hôpital une pleine année plus tôt qu'au début du siècle, et les garçons, presque deux ans plus tôt ${ }^{48}$. La principale cause de cette transition était d'ordre économique : la direction souhaitait réduire les dépenses en logeant les enfants de 12 ans et plus dans des familles d'accueil. Ces enfants étaient envoyés à la campagne, où ils intégraient le ménage de familles plus pauvres, le plus souvent chez des journaliers et des laboureurs. La plupart des villages accueillant ces enfants se trouvaient au nord-ouest de Dijon, dans l'Auxois et le Châtillonnais. Au cours d'une année donnée, des dizaines de petits villages, dont Grosbois (aujourd'hui Grosbois-en-Montagne), Bligny-le-Sec, Salmaise, Sombernon et Trouhaut, accueillaient régulièrement des centaines d'enfants et recevaient des milliers de livres envoyés par l'hôpital de Dijon.

À mesure que vieillissaient les enfants, ces derniers étaient idéalement en mesure de contribuer aux économies du foyer d'accueil tout en s'intégrant au tissu social du village. À Dijon, la direction comptait sur les curés de la région pour attester de la qualité des familles d'accueil et de l'enseignement rudimentaire reçu à l'école du village. Les comptes-rendus de leurs délibérations 
nous apprennent que ces espoirs n'étaient pas toujours comblés. On savait que certains enfants se faisaient maltraiter. Quelques-uns devaient mendier dans la rue, d'autres ne faisaient pas de catéchisme ou n'étaient pas envoyés à l'école du village de novembre à mars comme il avait été convenu ${ }^{49}$. L'hôpital récupérait quelques-uns de ces enfants pour leur faire faire leur première communion. Beaucoup d'enfants avaient des besoins particuliers qui les rendaient incapables de contribuer par leur travail aux ressources du ménage au même âge que pouvait le faire un enfant valide. Certains souffraient d'une incapacité physique ou mentale, et leur état contraignait l'hôpital à surveiller plus longtemps leur développement ${ }^{50}$. Pourtant, les schémas de circulation et les signes d'une intégration sociale réussie, même dans les cas les plus difficiles, montrent que la doctrine in loco parentis était acceptée et que la notion d'adoption telle que nous la connaissons aujourd'hui était en voie d'émergence au XVIII ${ }^{\mathrm{e}}$ siècle en Bourgogne.

Les jeunes abandonnés à la petite enfance pouvaient s'attendre à vivre avec deux ou trois familles d'accueil, parfois plus, souvent dans des villages différents, avant l'âge de 12 ans. Selon le contrat d'accueil renouvelable, une famille qui accueillait chez elle un enfant de 6 à 12 ans recevait trois livres par mois pendant deux ou trois ans. En Bourgogne, un journalier gagnait à l'époque environ une livre par jour, ou 200 livres par année. La somme de 36 livres par année n’était donc pas dérisoire. Il n'était pas rare qu'un enfant retourne à l'hôpital après un séjour de trois ans dans une famille d'accueil pour être envoyé le lendemain même à une nouvelle famille dans un autre village. Parfois, cela se produisait malgré des problèmes de santé ou de comportement clairement identifiables. Même un enfant pour qui la stabilité n'était pas la norme aura trouvé une telle transition très difficile sur les plans émotif et psychologique. Quand un enfant fêtait ses 12 ans, sa famille d'accueil pouvait choisir de le garder, mais on présumait que l'enfant pouvait désormais contribuer à la bourse familiale de telle façon que l'hôpital n'aurait plus à verser de paiements à la famille. Un enfant incapable de travailler efficacement, que ce soit en raison d'un handicap physique ou à cause de problèmes de comportement, était souvent renvoyé tout de suite à l'hôpital ${ }^{51}$. Dans certains cas, le contrat stipulait qu'une somme précise devait être versée à l'enfant. Dans d'autres instances, la famille d'accueil d'un enfant atteint d'un handicap physique l'empêchant de contribuer efficacement à l'économie familiale continuait à recevoir un versement mensuel.

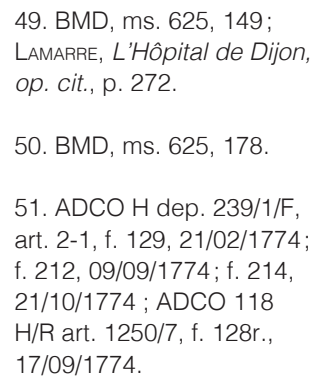


52. ADCO H dep. 239/1/F, art. 2-1, f. 121, 18/03/1770

53. ADCO, $118 \mathrm{H} / \mathrm{R}$ art. $1250 / 7$, f. $48 \mathrm{v}$., $11 / 11 / 1752$

54. $A D C O 118 \mathrm{H} / \mathrm{R}$ art. $1250 / 7$, f. $111 \mathrm{v}$., 24/01/1774

55. ADCO H dep. 239/1/F, art. 2-1, f. 130., 29/06/1774
Quand une famille d'accueil allait au-delà de ce que l'on attendait d'elle pour veiller au bien-être de l'enfant, l'hôpital pouvait augmenter le montant des versements mensuels ${ }^{52}$.

Malgré la nature quasi entrepreneuriale et le roulement rapide des situations d'accueil, des preuves de liens forgés entre les jeunes et leurs familles d'accueil abondent. En novembre 1752, Benoit de Coustant, 11 ans, rentrait d'un séjour dans une famille d'accueil à Quetigny, aux abords de Dijon. Il est resté chez les Bonnets Rouges jusqu'en mars de 1755, après quoi il a été confié à une nouvelle famille d'accueil à Sombernon. Cette famille a renouvelé son contrat d'accueil deux ans plus tard, en 1757, alors que Coustant avait 16 ans. Les contrats signés en 1755 et en 1757 donnaient droit à une somme de 15 livres par année, moins que la moitié de ce que l'hôpital versait normalement pour le soin d'un jeune enfant, mais l'établissement avait promis à la famille une somme de 60 livres après la première communion de Coustant ${ }^{53}$. Ce paiement, vers le milieu ou la fin de l'adolescence, était un signe d'intégration sociale et marquait la fin de la tutelle par l'hôpital. Les fonds pouvaient servir à payer une formation professionnelle, des vêtements, ou même des dépenses liées à un futur mariage. En l'occurrence, Coustant a probablement pu passer avec succès à la prochaine étape de sa vie grâce à l'appui que lui ont accordé la direction de l'hôpital et sa famille d'accueil.

Certains parents d'accueil ont voulu officialiser leur lien avec l'enfant dont ils se chargeaient en employant un langage technique et affectif dans leurs ententes formelles avec l'hôpital, lesquels ressemblaient aux contrats d'apprentissage déjà évoqués. Lorsqu'une famille acceptait de s'occuper d'un enfant comme s'il était le sien, l'enfant en question était parfois plus âgé. Nicolas Jean-Baptiste, par exemple, avait été abandonné à l'âge de huit jours et avait vécu toute sa vie dans des familles d'accueil et à l'hôpital. Il avait 16 ans quand Gaspard Dupatier a promis « d'en avoir soin comme de son propre enfant et de lui donner des gages proportionnés à ses services ${ }^{54}$ „. Charlotte Chapetot, quant à elle, est retournée vivre trois ans à Sainte-Anne après avoir logé dans une famille d'accueil à Salmaise. C'est alors qu'elle a été « confiée aux soins de Françoise Monin, femme de Philibert Perrier jardinier [...] qui a promis d'en avoir soin comme de son propre enfant soit pour le spirituel soit pour le temporal et encore de luy donner 18 livres de gages pour la première année ${ }^{55}$ ". 
S'il était techniquement interdit en France d'adopter un enfant, les familles et les directeurs des hôpitaux prêtaient à ce terme des usages qui seront familiers pour l'observateur moderne ${ }^{56}$. Catherine Gaulon et Anthoine Richard, par exemple, ont " adopté " Jean Gibassier, 9 ans ${ }^{57}$. D'autres cas où l'on parle d'adoption, certes peu communs, parsèment les registres ${ }^{58}$. L'expérience vécue par «Catherine » en 1754 sert de mise en garde dans l'éventualité où nous serions tentés d'assimiler ces expériences aux pratiques modernes. Ayant vécu presque toute sa vie dans des familles d'accueil, Catherine a été " adoptée " par un cordonnier à 17 ans. Moins d'un an et demi plus tard, cependant, les problèmes de santé de la fille ont contraint le cordonnier à l'abandonner à l'hôpital, où elle est morte peu de temps après ${ }^{59}$. Même des parents adoptifs pouvaient se départir de leurs enfants.

Sur une note plus encourageante, nous voyons d'autres signes d'une intégration sociale réussie en examinant des contrats d'accueil renouvelés avec différentes familles d'un même village. Ces documents historiques portent à croire que ces enfants avaient développé des liens sociaux importants à l'extérieur des confins de la famille d'accueil. C'était le cas, par exemple, de Joseph "Grosbois ", dont la famille d'accueil, dans le village de Grosbois, l'avait retourné à l'hôpital en novembre 1754. Peu de temps après, Joseph avait été renvoyé dans le même village, cette fois chez Joseph de la Borde, un manœuvrier. Sur le contrat, il est écrit que le jeune y serait « mis en pension [...] jusqu’à l'âge de 19 ans, au bout duquel temps luy sera donné un métier ${ }^{60}$ ». Denise, une enfant illégitime renvoyée à Sainte-Anne après avoir été mise en pension à Gissey-sur-Ouche en novembre 1753, est retournée dans ce même village un an et demi plus tard pour vivre dans une famille qui avait promis de « la garder gratuitement ${ }^{61}{ }^{\prime}$. De toute évidence, le temps que ces enfants avaient passé dans leur village d'accueil leur avait permis de se constituer un réseau d'amis et d'alliés en dehors de l'enceinte de leur foyer nourricier. La recirculation de ces enfants dans d'autres foyers leur épargnait parfois la nécessité de forger de nouveaux liens.

Certaines décisions prises par les enfants indiquent aussi que ces derniers ont réussi à s'intégrer dans le tissu social des villages bourguignons. Quelques-uns d'entre eux, des garçons surtout, ont tout fait pour maintenir les liens forgés dans leur village d'accueil. D'après les registres, le risque de fuite des jeunes était plus élevé juste après une transition importante, au moment de rentrer à

\author{
56. GAGER, Blood Ties and \\ Fictive Ties. \\ 57. ADCO $118 \mathrm{H} / \mathrm{R}$ \\ art. $1250 / 7$, f. 43 r. \\ 01/07/1751. \\ 58. ADCO H dep. 239/1/F, \\ art. 2-1, f. 72v., 11/05/1752 \\ f. 74 v., 13/09/1752; ADCO \\ $118 \mathrm{H} / \mathrm{R}$ art. $1250 / 7$, f. \\ 55v., 22/07/1754; 56v., \\ $15 / 09 / 1754$ et $04 / 10 / 1754$. \\ 59. ADCO H dep. 239/1/F, \\ art. 2-1, f. 83v, 07/09/1754. \\ 60. ADCO, $118 \mathrm{H} / \mathrm{R}$ \\ art. $1250 / 7$, f. 57r., \\ $11 / 11 / 1754$
}

61. ADCO, H dep. 239/1/F art. 2-1, f. 79r., 15/11/1753 
62. $\mathrm{ADCO} 118 \mathrm{H} / \mathrm{R}$ art. 1250/7, f. 57v., $10 / 11 / 1754$

63. Dinet-Lecomte, dans "Les enfants de la misère à l'hôpital », p. 215, rapporte que $14 \%$ des garçons et $1,4 \%$ des filles ont fui I'hôpital de Blois. Voir aussi Preux Bernard, Enfance abandonnée au XVIII siècle en Franche-Comté, Paris, L'Harmattan, 2014, p. 235.

Par contraste, Nelson constate qu'un faible pourcentage d'enfants (moins de

$3 \%$ ) avaient fui l'établissement dans la première moitié du XvıII siècle.

64. ADCO $118 \mathrm{H} / \mathrm{R}$ art. 1250/7, f. 119, 19/04/1772

65. ADCO $118 \mathrm{H} / \mathrm{R}$ art. $1250 / 7$, f. 54 v. et $59 v$., $15 / 04 / 1754$

66. ADCO, H dep. 239/1/F art. 2-1, f. 65v., 02/02/1750, et f. $66 r ., 27 / 06 / 1750$

67. Selon Dinet-LeCOMTE, "Les enfants de la misère à l'hôpital », p. 213, 52 \% des garçons et $42 \%$ des filles accueillis à Blois avaient fait une transition de l'hôpital vers la vie en société.

68. Beauvalet-Boutouyrie, "Les enfants de la Salpêtrière au xVIII ${ }^{e}$ siècle », p. 896 ; Dinet-LeCOMTE, ibid., p. 214.
Dijon ou de passer d'une maison ou d'un village à un autre. François, 12 ans, s'est enfui le lendemain de son retour à l'hôpital en novembre 1754 et n'a jamais été retrouvé ${ }^{62}$. Les données de 1750-1754 et 1770-1774 dont nous disposons montrent que $8 \%$ des enfants se sont enfuis de l'hôpital, cette action ayant été plus fréquente chez les garçons (13\% d'entre eux) que chez les filles $(2 \%)$. Selon des recherches menées sur l'hôpital de Blois, il s'agissait peut-être d'une tendance lourde à l'époque ${ }^{63}$. Parfois, les distances franchies par ces jeunes en fuite étaient remarquables. Nicolas, 18 ans, abandonné à l'hôpital pour la première fois à 11 ans, s'est rendu jusqu'à Lyon après s'être enfui, où il a été arrêté puis retourné à Dijon ${ }^{64}$. En retournant aux Bonnets Rouges à la fin d'un contrat d'accueil à Sombernon, Jean Baptiste dit St. George, 12 ans, a " trouvé un moyen de sortir et s'en retourner au lieu de Sombernon ", à environ 30 kilomètres à l'ouest de Dijon. On l'a retrouvé dans son ancienne famille d'accueil ${ }^{65}$. S’il est vrai que les garçons étaient plus aptes que les filles à s'enfuir, il arrivait tout de même qu'une fille tente sa chance. À l'occasion d'une promenade dominicale en juillet 1750, Pierrette Chamureau, 12 ans, et Reine Colibus, 9 ans, ont décidé de prendre la clé des champs. Pierrette a été retrouvée deux mois plus tard à 34 kilomètres de là, dans son village adoptif de Trouhaut, avant d'être renvoyée à Dijon où elle a été réprimandée. La plus jeune des deux, de toute évidence plus astucieuse que son aînée, a réussi à échapper à la direction de l'hôpital jusqu'en octobre 1751, plus d'un an après avoir fui Dijon ${ }^{66}$.

Comme le laissent entendre les données relatives aux jeunes fugueurs, les registres ne peuvent donner qu'un aperçu partiel de l'intégration sociale des jeunes. Entre un quart et un tiers des entrées ne révèlent rien de l'avenir des enfants dont il est question. Du reste, l'information contenue dans ces registres donne à entendre que près d'un enfant sur dix est mort à l'adolescence pendant que l'hôpital s'en occupait (8\% des garçons et $11 \%$ des filles). Qui plus est, un peu plus de la moitié des jeunes ( $54 \%$ des filles et $57 \%$ des garçons) auront connu ce que l'on pourrait appeler une intégration sociale réussie au moment de leur transition vers la fin de l'adolescence et le début de l'âge adulte ${ }^{67}$. Durant la seconde moitié du siècle, un parent ou un autre membre de la famille est venu récupérer l'enfant dans environ $5 \%$ des cas [un chiffre appréciablement moins élevé qu'à la Salpêtrière de Paris $(62,7 \%)$ ou à l'hôpital de Blois $(46 \%)]^{68}$. En tout, $38 \%$ des garçons et $31 \%$ des filles ont été replacés à la campagne dans des familles d'accueil pendant un à trois ans après avoir été admis à l'hôpital vers 
l'âge de 12 ans. S'ils survivaient, la plupart d'entre eux allaient à la campagne et travaillaient comme ouvriers agricoles, laboureurs ou domestiques dans leur village d'adoption en Bourgogne.

Un plus petit nombre d'enfants ( $15 \%$ des garçons et $18 \%$ des filles) se voyaient confier un métier. De façon générale, les jeunes filles trouvaient un emploi dans le secteur des services, la vaste majorité d'entre elles en service domestique ou comme blanchisseuses. Un plus petit nombre de filles réussissaient à se trouver un emploi dans le commerce textile, comme couturières surtout. Les jeunes hommes travaillaient comme apprentis auprès d'un maître cordonnier, menuisier, ébéniste, serrurier, maçon ou outilleur, autant de métiers qui n'exigeaient pas une longue formation coûteuse ${ }^{69}$. Quelques jeunes, comme Jean-Baptiste Mathieu, quittaient l'hôpital en espérant pouvoir forger euxmêmes leur avenir. Au moment d'être renvoyé à l'hôpital par sa famille d'accueil en avril 1773, Mathieu avait 15 ans. En sept mois, il s'est enfui deux fois avant d'être renvoyé à l'hôpital par les autorités en novembre de la même année. Deux mois plus tard, en janvier 1774, le jeune homme « est reparti de son plein gré pour chercher un maître, disant qu'il ne pouvait se plaire à l'hôpital ${ }^{70}$ ».

\section{ConClusion}

Comme l'ont montré Kristen Gager et Matthew Gerber, un nombre grandissant de critiques sociaux ont milité pour des changements dans la seconde moitié du XVIII ${ }^{\mathrm{e}}$ siècle qui auront permis l'adoption de règles plus souples en matière d'héritage et une meilleure intégration sociale d'enfants abandonnés ou illégitimes. Le nombre croissant d'enfants abandonnés était à la racine du problème, puisque ces derniers posaient un grave dilemme financier et moral aux organismes de bienfaisance et à l'État. La solution a été de réintégrer ces enfants dans des familles sans tenir compte de leur appartenance ou de leur lignée. Au début des années 1780, Louis-Sébastien Mercier écrivait, en parlant de Paris, que « la foule de nécessiteux augmentant chaque jour en proportion du nombre des riches, une loi qui établiroit l'adoption seroit sans doute une des plus utiles qu'on pût faire aujourd'hui en France ${ }^{71}$ ». La recirculation, l'intégration sociale et parfois même l'adoption d'enfants en Bourgogne et en France prouvent que l'Église et l'État parrainaient des affiliations loco parentis ad hoc et non biologiques, de telle façon à remettre en question, ironiquement, la façon dont l'État concevait l'autorité parentale et la composition familiale.
69. Cette tendance est à peu près la même pour l'ensemble de la France. Voir Beauvalet-Boutouyrie, op. cit., p. 896 ; DINETLECOMTE, op. cit., p. 214; GutTon, « L'insertion sociale des enfants recueillis par la charité de Lyon au xvIII siècle », p. 932; Lamarre, «La vie des enfants et des vieillards... ", op. cit., p. 34 ; et Nelson, "Gender, Age... », op. cit., p. 126-127.

70. ADCO $118 \mathrm{H} / \mathrm{R}$ art. $1250 / 7$, f. $125 \mathrm{v}$., 06/04/1773.

71. Mercier Louis-Sébastien, Tableau de Paris, tome 7, Paris, 1783 , p. 577 , cité dans Gager, Blood Ties and Fictive Ties, p. 164. 
72. Gerber, Bastards..., op. cit., p. 133-155.

73. Pour en savoir plus sur la perspective traditionnelle sur les modèles familiaux au début de l'époque moderne, voir LASLETT Peter et WALL Richard, Household and Family in Past Time,

Cambridge, Cambridge University Press, 1972.

Vers la fin de sa carrière, Laslett remettait lui-même en question l'utilité des modèles structurels. À ce sujet, voir par exemple « Family, Kinship and Collectivity as Systems of Support in Pre-Industrial Europe ", Continuity and Change, 3, 2, 1988, p. 153-176.
Créées et adaptées par nécessité, ces pratiques ad hoc servaient l'intérêt public en proposant un moyen ordonné et rentable d'intégrer des jeunes dans la société à mesure qu'ils grandissaient ${ }^{72}$.

Même si ces démarches n'étaient pas reconnues par la loi, elles étaient soutenues par des coutumes populaires qui permettaient de faire circuler des enfants et des jeunes dans des foyers où ils étaient élevés par des gens autres que leurs parents, souvent loin de chez eux, et fréquentaient des écoles, des ateliers ou des orphelinats. Si l'idéal social et juridique continuait d'être axé sur le patriarche, il arrivait souvent que des personnes de la communauté se chargent d'élever les jeunes de Dijon. Et s'il est vrai que les familles agricoles, en milieu rural, auraient préféré garder leurs enfants près d'eux le plus longtemps possible afin que ces derniers puissent contribuer aux activités du ménage, il est vrai aussi que les jeunes quittaient souvent la maison dès un jeune âge pour acquérir des compétences et l'expérience dont ils auraient besoin pour s'intégrer socialement. La circulation des enfants n'était pas réservée aux riches : même l'économie de fortune des pauvres savait reconnaître les avantages de faire circuler ainsi les jeunes. Un chef de ménage en difficulté était parfois contraint d'envoyer ses enfants à une institution de bienfaisance pendant un certain temps; dans certains cas, c'était de manière plus permanente. Cette institution pouvait à son tour faire circuler ces enfants dans des foyers d'accueil à la campagne, leur offrir une éducation de base et, dans certains cas, les aider à atteindre un certain degré d'autonomie en les plaçant comme apprentis au sein d'un réseau. La circulation et la recirculation des jeunes était une composante importante du système familial de l'ère moderne plutôt qu'une exception à la règle ou une aberration des " systèmes familiaux " traditionnels qu' exposaient les historiens démographes des années 1960 et $1970^{73}$. À la manière de la tutelle, elle nous permet d'observer la souplesse et la faculté d'adaptation des premières familles modernes. En reconnaissant l'existence de ces pratiques, nous gagnons une perspective beaucoup plus large sur les modèles de ménage " alternatifs » et les dimensions pratiques de l'autorité familiale en France au début de l'époque moderne. 